\title{
OSCILLATION ANALYSIS OF NUMERICAL SOLUTION FOR BLOOD CELL PRODUCTION MODEL
}

\author{
QI WANG*, QIAN YANG, JIEYI YAO \\ School of Applied Mathematics, Guangdong University of Technology, Guangzhou, China \\ *Corresponding author: bmwzwq@126.com \\ Received May 3, 2020
}

\begin{abstract}
AвSTRACt. The paper deals with the numerical oscillation for a nonlinear delay differential equation that represents a blood cell production model. Some conditions under which the numerical solution oscillates are established. Meanwhile, it is proven that the non-oscillatory numerical solution tends to the unique positive fixed point of the original continuous-time system. Numerical simulations are given to illustrate the theoretical findings. The results in this paper extend the former ones from the view point of numerical meaning.
\end{abstract}

2010 Mathematics Subject Classification. 65L07; 65L20.

Key words and phrases. Nonlinear delay differential equation; Numerical solution; Oscillation.

\section{InTRODUCTION}

In this paper, we consider the following nonlinear delay differential equation (DDE)

$$
p^{\prime}(t)=\frac{\beta p^{a}(t-\tau)}{1+p^{b}(t-\tau)}-\gamma p(t),
$$

with positive constants $\beta, \gamma, \tau$ and $a, b \in \mathbb{N}$.

Eq. (1.1) was proposed as model of blood cell production where $p(t)$ denotes the density of mature cells in blood circulation and $\tau$ is the time between the production of immature cells in the bone marrow and their maturation for release in the circulating bloodstreams. The flux $f(p(t-\tau))=\left(\beta p^{a}(t-\tau)\right) /\left(1+p^{b}(t-\tau)\right)$ of the cells into the circulation from the stem cell compartment depends on $p(t-\tau)$ at time $t-\tau$. In the sense of the work of Mackey and Nechaeva [14], in (1.1), if $0=a<b$, the feedback is negative, if $0<a<b$, mixed, while $0<a=b$, positive. When $a=0$, (1.1) leads to the hematopoiesis model which was first 
proposed by Mackey and Glass [15] to describe some physiological control systems. Also, when $a=1$, (1.1) reduces to the well-known "classical" Mackey-Glass equation [15]. Moreover, a positive equilibrium point $\bar{p}$ satisfies $\beta(\bar{p})^{a} /\left(1+(\bar{p})^{b}\right)=\gamma \bar{p}$. In the special case where $b>a$ and there is a unique positive equilibrium point $\bar{p}$.

Recently, many results on (1.1) have been widely discussed. When $a=0$, El-Sheikh et al. [4] obtained conditions for oscillation and global attractivity of (1.1). Later, Saker [16] extended and improved the corresponding results given in [4] for general $a$ and $b$. When $a=1$, Hale and Sternberg [10] gave interesting and nice results for the numerical and chaotic problems of (1.1). For more information on (1.1), we refer the reader to [13] and the references cited therein. However, to the best of our knowledge, the former results are all on analytical aspects, numerical behaviors have been considered rarely. Our aim in this work is to consider both numerical oscillation and non-oscillation of (1.1). The main contribution of this paper is that we get the sufficient condition under which the numerical solutions oscillate. Meanwhile, asymptotic behavior of non-oscillatory numerical solutions is discussed also. So they enrich the application of numerical methods.

The property of oscillation is one of the most concerns in the field of differential equation. Recently, it has received much attention from a number of investigators $[1,3,5-7,11,12,19]$. The following results are useful for the subsequent analysis.

Theorem 1. ( [8]) Consider the following equation

$$
a_{n+1}-a_{n}+\sum_{j=-k}^{l} q_{j} a_{n+j}=0,
$$

assume $k, l \in \mathbb{N}$ and $q_{j} \in \mathbb{R}$ for $j=-k, \cdots, l$, then the following two statements are equivalent: $(a)$ Every solution of (1.2) oscillates; (b) The characteristic equation $\lambda-1+\sum_{j=-k}^{l} q_{j} \lambda^{j}=0$ has no positive roots.

Theorem 2. ( [8]) For the equation

$$
a_{n+1}-a_{n}+p a_{n-k}+q a_{n}=0,
$$

where $k, p, q>0$, the necessary and sufficient conditions for the oscillation of $(1.3)$ are $q \in(0,1)$ and $p(k+1)^{k+1} / k^{k}>(1-q)^{k+1}$.

Theorem 3. ( [16]) If $b>a$, then every solution of (1.1) oscillates about $\bar{p}$ if and only if

$$
Q e^{\gamma \tau} \tau>\frac{1}{e}
$$


where $Q=\left(\beta(b-a)(\bar{p})^{b+a-1}-\beta a(\bar{p})^{a-1}\right) /\left(\left(1+(\bar{p})^{b}\right)^{2}\right)$.

\section{THE DISCRETE SCHEME}

Firstly, we introduce an invariant oscillation transformation to (1.1), then apply the corresponding numerical method to this type.

After a transform $p(t)=\bar{p}+z(t),(1.1)$ can be changed into

$$
z^{\prime}(t)+\gamma f_{1}(z(t))+Q f_{2}(z(t-\tau))=0
$$

where

$$
f_{1}(u)=u
$$

and

$$
f_{2}(u)=\frac{\left(1+(\bar{p})^{b}\right)^{2}}{(b-a)(\bar{p})^{b+a-1}-a(\bar{p})^{a-1}}\left(\frac{(\bar{p})^{a}}{1+(\bar{p})^{b}}-\frac{(\bar{p}+u)^{a}}{1+(\bar{p}+u)^{b}}\right)
$$

satisfy

$$
\begin{array}{lll}
u f_{1}(u)>0, & \text { for } \quad u \neq 0, & \lim _{u \rightarrow 0} f_{1}(u) / u=1, \\
u f_{2}(u)>0, & \text { for } \quad u>0, & \lim _{u \rightarrow 0} f_{2}(u) / u=1 .
\end{array}
$$

Obviously, $p(t)$ oscillates about $\bar{p}$ if and only if $z(t)$ oscillates.

Let $h=\tau / m$ be the step size, $m$ is a positive integer, application of the $\theta$-methods $(0 \leq \theta \leq 1)$ to $(2.1)$, we get

$$
z_{n+1}=z_{n}-h \theta \gamma f_{1}\left(z_{n+1}\right)-h \theta Q f_{2}\left(z_{n+1-m}\right)-h(1-\theta) \gamma f_{1}\left(z_{n}\right)-h(1-\theta) Q f_{2}\left(z_{n-m}\right),
$$

where $z_{n+1}$ and $z_{n+1-m}$ are approximations to $z(t)$ and $z(t-\tau)$ of $(2.1)$ at $t_{n+1}$, respectively.

Let $z_{n}=p_{n}-\bar{p}$, we obtain

$$
p_{n+1}=\frac{1-h(1-\theta) \gamma}{1+h \theta \gamma} p_{n}+\frac{h \theta \beta}{1+h \theta \gamma} \frac{p_{n+1-m}^{a}}{1+p_{n+1-m}^{b}}+\frac{h(1-\theta) \beta}{1+h \theta \gamma} \frac{p_{n-m}^{a}}{1+p_{n-m}^{b}}
$$

By the Method of Steps [9], we can get the following result.

Theorem 4. The $\theta$-methods given by (2.4) is convergent with order $1(\theta \neq 0.5)$ and $2(\theta=0.5)$. 


\section{Oscillation OF NUMERICAL SOLUTION}

It is clear that $p_{n}$ oscillates about $\bar{p}$ if and only if $z_{n}$ is oscillatory. In order to investigate oscillation of (2.4), we only need to study the oscillation of (2.3). Eq. (2.3) can be linearized to

$$
z_{n+1}=z_{n}-h \theta \gamma z_{n+1}-h \theta Q z_{n+1-m}-h(1-\theta) \gamma z_{n}-h(1-\theta) Q z_{n-m},
$$

so

$$
z_{n+1}-R(-\gamma h) z_{n}+\frac{Q \theta h}{1+Q \gamma h} z_{n+1-m}+\frac{Q(1-\theta) h}{1+Q \gamma h} z_{n-m}=0,
$$

where $R(x)=(1+(1-\theta) x) /(1-\theta x)$ is the stability function (see [2]) of the $\theta$-methods. It follows from [12] that (2.3) oscillates if (3.2) oscillates under the condition (2.2).

Next, we will investigate whether the $\theta$-method preserve the oscillation of (1.1). That is, when Theorem 3 holds, we will study the conditions under which (2.4) is oscillatory.

Lemma 1. The characteristic equation of (3.1) is given by

$$
\lambda=R\left(-h\left(\gamma+Q \lambda^{-m}\right)\right) .
$$

Proof: From (3.1) and the expression of $R(x)$ we can get this proof.

Lemma 2. If Theorem 3 holds, then (3.3) has no positive roots for $0 \leq \theta \leq 0.5$.

Proof: Set $A(\lambda)=\lambda-R\left(-h\left(\gamma+Q \lambda^{-m}\right)\right)$. From Lemma 3 in [17] we know

$$
R\left(-h\left(\gamma+Q \lambda^{-m}\right)\right) \leq \exp \left(-h\left(\gamma+Q \lambda^{-m}\right)\right) .
$$

Next we prove that $B(\lambda)=\lambda-\exp \left(-h\left(\gamma+Q \lambda^{-m}\right)\right)>0$ for $\lambda>0$. Suppose there is $\lambda_{0}>0$ such that $B\left(\lambda_{0}\right) \leq 0$, then $\lambda_{0} \leq \exp \left(-h\left(\gamma+Q \lambda_{0}^{-m}\right)\right)$ and

$$
\lambda_{0}^{m} \leq \exp \left(-\gamma \tau-Q \tau \lambda_{0}^{-m}\right) .
$$

Further we have

$$
Q \tau e^{\gamma \tau} e \leq Q \tau \lambda_{0}^{-m} \exp \left(1-Q \tau \lambda_{0}^{-m}\right),
$$

so we get the following two cases:

(i) If $1-Q \tau \lambda_{0}^{-m}=0$, then $Q \tau e^{\gamma \tau} e \leq 1$, this contradicts with (1.4).

(ii) If $1-Q \tau \lambda_{0}^{-m} \neq 0$, then

$$
\exp \left(1-Q \tau \lambda_{0}^{-m}\right)<\frac{1}{1-\left(1-Q \tau \lambda_{0}^{-m}\right)}=\frac{1}{Q \tau \lambda_{0}^{-m}},
$$


equivalently

$$
Q \tau \lambda_{0}^{-m} \exp \left(1-Q \tau \lambda_{0}^{-m}\right)<1
$$

that is

$$
Q \tau e^{\gamma \tau} e<1
$$

which is also a contradiction to (1.4). Thus

$$
A(\lambda)=\lambda-R\left(-h\left(\gamma+Q \lambda^{-m}\right)\right) \geq \lambda-\exp \left(-h\left(\gamma+Q \lambda^{-m}\right)\right)=B(\lambda)>0
$$

which implies that (3.3) has no positive roots. This ends the proof.

Next we assume that $m>1$ in the case of $0.5<\theta \leq 1$.

Lemma 3. If Theorem 3 holds and $0.5<\theta \leq 1$, then (3.3) has no positive roots for $h<\bar{h}$, where

$$
\bar{h}=\left\{\begin{array}{l}
\infty, \quad \text { if } \quad Q \tau \geq 1 \\
\frac{\tau(1+\gamma \tau+\ln Q \tau)}{1+\gamma \tau(1-\ln Q \tau)}, \quad \text { if } \quad Q \tau<1
\end{array}\right.
$$

Proof: Since $R\left(-h\left(\gamma+Q \lambda^{-m}\right)\right)$ is increasing about $\theta$ when $\lambda>0$, then

$$
R\left(-h\left(\gamma+Q \lambda^{-m}\right)\right)=\frac{1-h(1-\theta)\left(\gamma+Q \lambda^{-m}\right)}{1+h \theta\left(\gamma+Q \lambda^{-m}\right)} \leq \frac{1}{1+h\left(\gamma+Q \lambda^{-m}\right)} .
$$

From now on, we will prove that

$$
\lambda-\frac{1}{1+h\left(\gamma+Q \lambda^{-m}\right)}>0
$$

holds under some conditions.

Denote

$$
\omega(\lambda)=\lambda^{m}-\frac{1}{1+h \gamma} \lambda^{m-1}+\frac{h Q}{1+h \gamma},
$$

rearrange the left side of (3.7) gives

$$
\lambda-\frac{1}{1+h\left(\gamma+Q \lambda^{-m}\right)}=\frac{(1+\gamma h) \lambda^{1-m}}{1+h\left(\gamma+Q \lambda^{-m}\right)} \omega(\lambda),
$$

next we will show that $\omega(\lambda)>0$ for $\lambda>0$. Obviously, $\omega(\lambda)$ is the characteristic polynomial of difference equation

$$
y_{n+1}-y_{n}+\frac{h \gamma}{1+h \gamma} y_{n}+\frac{h Q}{1+h \gamma} y_{n+1-m}=0 .
$$

From Theorems 1 and 2, we know that $\omega(\lambda)$ has no positive roots if and only if

$$
\frac{h Q}{1+h \gamma} \frac{m^{m}}{(m-1)^{m-1}}>\left(1-\frac{h \gamma}{1+h \gamma}\right)^{m},
$$


which can be transform to

$$
\ln Q \tau+(m-1) \ln \left(1+\frac{1+\gamma \tau}{m-1}\right)>0
$$

Thus, we have two possible cases to consider.

Case one, if $Q \tau \geq 1$, in view of $m>1$, then (3.8) holds true.

Case two, if $Q \tau<1$ and $h<(\tau(1+\gamma \tau+\ln Q \tau)) /(1+\gamma \tau(1-\ln Q \tau))$, then we get

$$
\begin{aligned}
& \ln Q \tau+(m-1) \ln \left(1+\frac{1+\gamma \tau}{m-1}\right) \\
> & \ln Q \tau+(m-1) \frac{\frac{1+\gamma \tau}{m-1}}{1+\frac{1+\gamma \tau}{m-1}}=\ln Q \tau+\frac{(m-1)(1+\gamma \tau)}{m+\gamma \tau}>0 .
\end{aligned}
$$

So (3.7) holds for $h<\bar{h}$, where $\bar{h}$ is defined in (3.6). Therefore

$$
A(\lambda)=\lambda-R\left(-h\left(\gamma+Q \lambda^{-m}\right)\right)>\lambda-\frac{1}{1+h\left(\gamma+Q \lambda^{-m}\right)}>0
$$

holds for $h<\bar{h}$, which implies that (3.3) has no positive roots. The proof is completed.

By employing (2.2), Lemmas 2, 3 and Theorem 1, we have the first main result of this paper.

Theorem 5. If Theorem 3 holds, then (2.4) is oscillatory for $h<\infty$ when $0 \leq \theta \leq 0.5$ and $h<\bar{h}$ when $0.5<\theta \leq 1$, where $\bar{h}$ is defined in (3.6).

\section{Asymptotic BeHAVIOR OF NON-OSCILlatory NUMERICAL SOLUTION}

This section is devoted to the asymptotic property of non-oscillatory solution of (2.4).

Lemma 4. ( [16]) If $b>a$, then the positive solution of (1.1) which does not oscillate about $\bar{p}$ satisfies $\lim _{t \rightarrow \infty} p(t)=\bar{p}$.

We can see from (1.1) and (2.1) that the non-oscillatory solution of (2.1) satisfies $\lim _{t \rightarrow \infty} z(t)=0$ if Lemma 4 holds. Further, we will prove that the numerical solution of (1.1) can preserve this property.

Lemma 5. Let $z_{n}$ be a non-oscillatory solution of (2.3), then $\lim _{n \rightarrow \infty} z_{n}=0$.

Proof: In general, assume that $z_{n}>0$ for sufficiently large $n$. Then $f_{1}\left(z_{i}\right)>0$ and $f_{2}\left(z_{i}\right)>0$ for large $i$. It follows from (2.3) that

$$
\begin{aligned}
& z_{n+1}-z_{n}+h \theta Q f_{2}\left(z_{n+1-m}\right)+h(1-\theta) Q f_{2}\left(z_{n-m}\right) \\
= & -h \theta \gamma f_{1}\left(z_{n+1}\right)-h(1-\theta) \gamma f_{1}\left(z_{n}\right)<0,
\end{aligned}
$$

hence $z_{n+1}-z_{n}<0$, then $\left\{z_{n}\right\}$ is decreasing. Thus, there exists an $\eta \geq 0$ such that

$$
\lim _{n \rightarrow \infty} z_{n}=\eta
$$


We claim that $\eta=0$. If $\eta>0$, then there exists $N_{0} \in \mathbb{N}$ and $\epsilon>0$ such that for $n-m>N_{0}$, $0<\eta-\epsilon<z_{n}<\eta+\epsilon$. So $z_{n-m}>\eta-\epsilon$ and $z_{n+1-m}>\eta-\epsilon$, then (4.1) gives

$$
z_{n+1}-z_{n}+h \theta Q f_{2}(\eta-\epsilon)+h(1-\theta) Q f_{2}(\eta-\epsilon)<0,
$$

which implies that $z_{n+1}-z_{n}<L<0$, where

$$
L=h \beta\left(\frac{(\bar{p}+\eta-\epsilon)^{a}}{1+(\bar{p}+\eta-\epsilon)^{b}}-\frac{(\bar{p})^{a}}{1+(\bar{p})^{b}}\right) .
$$

So $z_{n} \rightarrow-\infty$ as $n \rightarrow \infty$, which is a contradiction to (4.2). The proof is finished.

Then the second main result of this paper is straightforward.

Theorem 6. Let $p_{n}$ be a positive solution of (2.4), which does not oscillate about $\bar{p}$, then $\lim _{n \rightarrow \infty} p_{n}=\bar{p}$.

Remark 1. If $a=0$, then (1.1) becomes

$$
p^{\prime}(t)=\frac{\beta}{1+p^{b}(t-\tau)}-\gamma p(t), \quad t \geq 0,
$$

which is the model of control of erythropoiesis [2]

$$
x^{\prime}(t)=\frac{\beta \rho^{n}}{\rho^{n}+x^{n}(t-\tau)}-\gamma x(t), \quad t \geq 0
$$

with $\rho=1$. The oscillation of numerical solution for (4.3) has been discussed in [18], so the results in [18] are the special cases of the corresponding ones in this paper.

\section{Numerical eXAmples}

In this section, we will present some numerical examples to check the validity of our results. Firstly, we consider the problem

$$
\begin{aligned}
& p^{\prime}(t)=\frac{3 p(t-4)}{1+p^{2}(t-4)}-p(t), \\
& p(t)=1.25, \quad-4 \leq t \leq 0 .
\end{aligned}
$$

It is easy to see that (1.4) holds true. That is, the analytic solution of (5.1) is oscillatory. Let $m=40$ and $\theta=0.2 \in[0,0.5]$ in FIGURE 1 . From this figure, we can see that the numerical solution of (5.1) oscillates about the positive equilibrium point $\bar{p}=\sqrt{2}$, which is in agreement with Theorem 5.

Secondly, for the problem

$$
\begin{aligned}
& p^{\prime}(t)=\frac{3.6 p(t-5)}{1+p^{2}(t-5)}-0.3 p(t), \\
& p(t)=4.1, \quad-5 \leq t \leq 0 .
\end{aligned}
$$


We check that (1.4) is valid. Equivalently, the analytic solution of (5.2) is oscillatory. Let $m=100$ and $\theta=0.7 \in[0.5,1]$ in FIGURE 2. By direct calculation we have $Q \tau=1.25 \geq 1$ and $h=0.05<\bar{h}=\infty$. We can also see that the numerical solution of (5.2) oscillates about $\bar{p}=\sqrt{11}$, which coincides with Theorem 5 .

Thirdly, we consider the following problem

$$
\begin{aligned}
& p^{\prime}(t)=\frac{2.5 p(t-2)}{1+p^{2}(t-2)}-0.5 p(t), \\
& p(t)=1.5, \quad-2 \leq t \leq 0 .
\end{aligned}
$$

One can easily get that the analytic solution of (5.3) is oscillatory. Set $m=20$ and $\theta=0.8 \in$ $[0.5,1]$ in FIGURE 3, so $Q \tau=0.6<1$ and $h=\tau / m=0.1<\bar{h}=1.1862$. We can see from FIGURE 3 that the numerical solution of (5.3) oscillates about $\bar{p}=2$, which is consistent with Theorem 5.

Finally, consider another problem

$$
\begin{aligned}
& p^{\prime}(t)=\frac{6.3 p(t-0.3)}{1+p^{2}(t-0.3)}-0.63 p(t), \\
& p(t)=10, \quad-0.3 \leq t \leq 0 .
\end{aligned}
$$

We easily obtain that (1.4) is not satisfied, so the analytic solution of (5.4) is non-oscillatory. Let $m=30$ and $\theta=0.4$ in FIGURE 4 , we can see that $p(t)$ and $p_{n}$ are both approach to equilibrium point 3, which coincides with Theorem 6.

In a word, we can see from these figures that the $\theta$-methods preserve the oscillation of (5.1) -(5.3) and the non-oscillation of (5.4), respectively.

\section{ACKNOWLEDGEMENTS}

This research is supported by the Natural Science Foundation of Guangdong Province (No. 2017A030313031). 

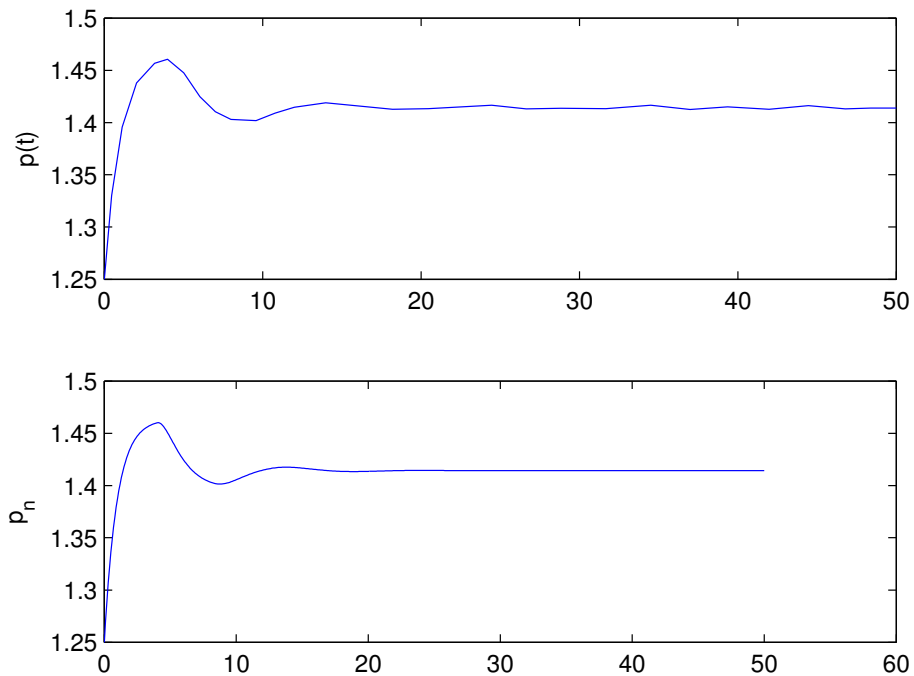

FIGURE 1. The analytic solution and the numerical solution of (5.1) with $\theta=0.2$ and $m=40$.
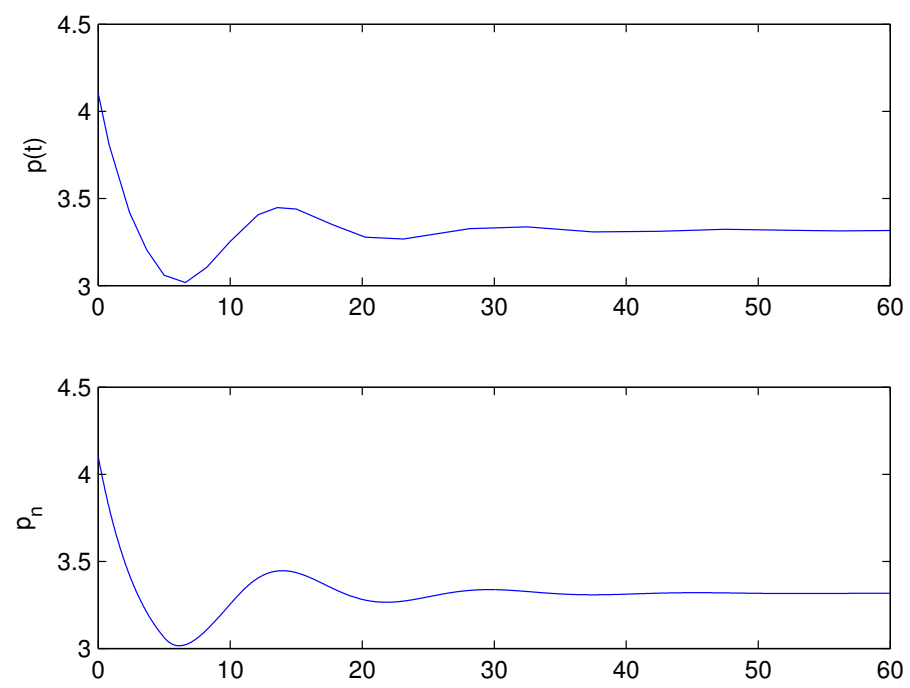

FIGURE 2. The analytic solution and the numerical solution of (5.2) with $\theta=0.7$ and $m=100$. 

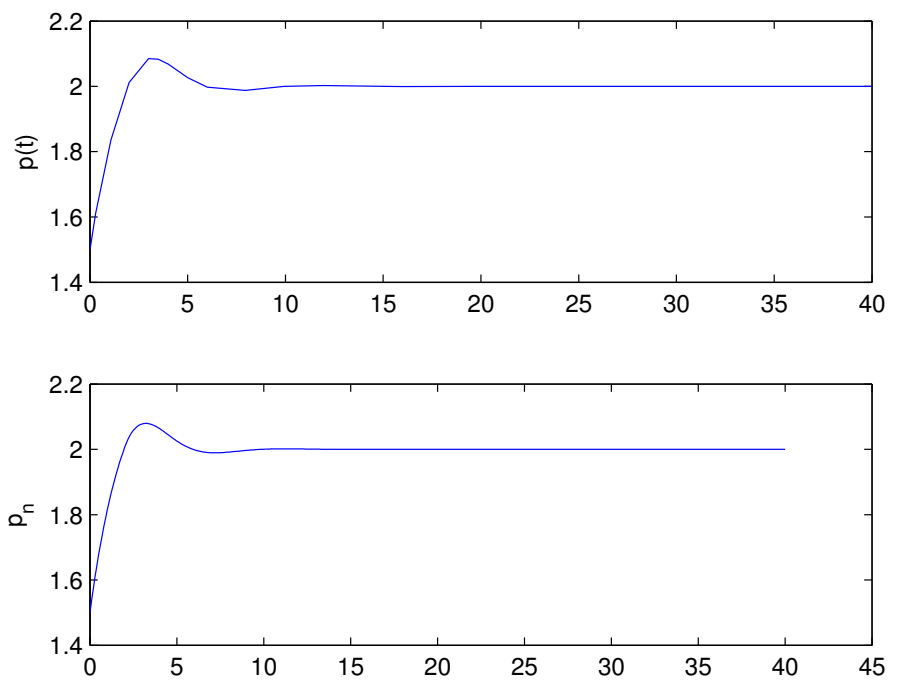

FIGURE 3. The analytic solution and the numerical solution of (5.3) with $\theta=0.8$ and $m=20$.
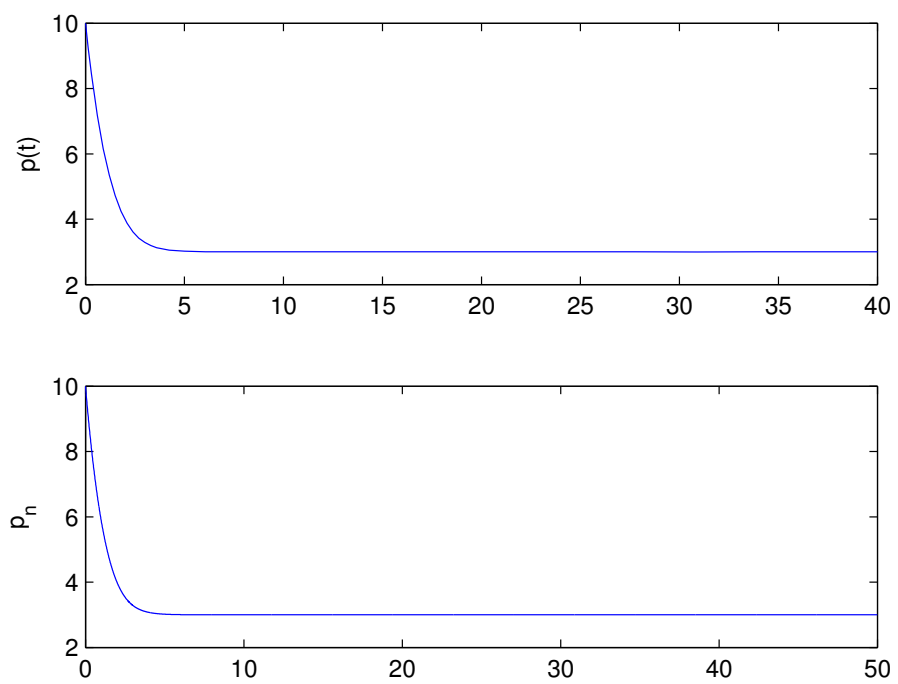

FIGURE 4. The analytic solution and the numerical solution of (5.4) with $\theta=0.4$ and $m=30$. 


\section{REFERENCES}

[1] G.E. Chatzarakis, Oscillation test for linear deviating differential equations, Appl. Math. Lett. 98 (2019), 352-358.

[2] K. Dekker, J.G. Verwer, Stability of Runge-Kutta methods for stiff nonlinear differential equations, NorthHolland, Amsterdam, 1984.

[3] J. Dzurina, Oscillatory behavior of the second order noncanonical differential equations, Appl. Math. Lett. 73 (2017), 62-68.

[4] M.M.A. El-Sheikh, A. Zaghrout, A. Ammar, Oscillation and global attractivity in delay equation of population dynamics, Appl. Math. Comput. 77 (1996), 195-204.

[5] H.A. El-Morshedy, E.R. Attia, New oscillation criterion for delay differential equations with non-monotone arguments, Appl. Math. Lett. 54 (2016), 54-59.

[6] K. Gopalsamy, Stability and Oscillations in Delay Differential Equations of Population Dynamics, Kluwer, The Netherlands, 1992.

[7] S.R. Grace, I. Jadlovska, A. Zafer, On oscillation of third-order noncanonical delay differential equations, Appl. Math. Comput. 362 (2019), 124530.

[8] I. Györi, G. Ladas, Oscillation Theory of Delay Differential Equations with Applications, Academic Press, Oxford, 1991.

[9] J. Hale, Theory of Functional Differential Equations, Springer-Verlag, New York, 1977.

[10] J. Hale, N. Sternberg, Onset of chaos in differential delay equations, J. Comput. Phys. 77 (1988), 221-239.

[11] T.X. Li, Yu.V. Rogovchenko, Oscillation criteria for even-order neutral differential equations, Appl. Math. Lett. 61 (2016), 35-41.

[12] T.X. Li, Yu.V. Rogovchenko, Oscillation criteria for second-order superlinear Emden-Fowler neutral differential equations, Monatsh. Math. 184 (2017), 489-500.

[13] B.W. Liu, New results on the positive almost periodic solutions for a model of hematopoiesis, Nonlinear Analysis: Real World Applications 17 (2014), 252-264.

[14] M.C. Mackey, I. Nechaeva, Noise and stability in differential delay equations, J. Dynam. Differential Equations 6 (1994), 395-426.

[15] M.C. Mackey, L. Glass, Oscillation and chaos in physiological control systems, Science 197 (1977), 287-289.

[16] S.H. Saker, Oscillation and global attractivity in hematopoiesis model with delay time, Appl. Math. Comput. 136 (2003), 241-250.

[17] M.H. Song, Z.W. Yang, M.Z. Liu, Stability of $\theta$-methods for advanced differential equations with piecewise continuous arguments, Comput. Math. Appl. 49 (2005), 1295-1301.

[18] Q. Wang, J.C. Wen, Oscillations of numerical solutions for nonlinear delay differential equations in the control of erythropoiesis, Discrete Dynamics in Nature and Society 2013 (2013), 1-9.

[19] Q. Wang, J.Y. Yao, Numerical stability and oscillation of a kind of functional differential equations, J. Liaocheng Univ. (Nat. Sci.) 33 (2020), 18-27. 